\title{
Spectroscopic studies of OB stars in the Magellanic Clouds with VLT-UVES
}

\author{
Christopher J. Evans, Paul A. Crowther \\ Department of Physics and Astronomy, University College London, \\ Gower Street, London WC1E 6BT, UK
}

\author{
Alexander W. Fullerton \\ Department of Physics and Astronomy, The Johns Hopkins University, \\ 3400 North Charles Street, Baltimore, MD 21286, USA \\ D. John Hillier \\ Department of Physics and Astronomy, University of Pittsburgh, \\ 3941 O'Hara Street, Pittsburgh, PA 15260, USA
}

\begin{abstract}
We present results from optical and ultraviolet analysis of nine LMC/SMC supergiants. Temperatures, mass-loss rates and $\mathrm{CNO}$ abundances are obtained using the non-LTE, line-blanketed model atmosphere code of Hillier \& Miller (1998). In general, the derived temperatures are significantly lower than those determined from unblanketed, plane-parallel models.
\end{abstract}

\section{Introduction}

The proximity of the Magellanic Clouds provides an excellent opportunity to study individual $\mathrm{OB}$ stars in metal-poor environments at both high resolution and high signal-to-noise, using the UV-Visual Echelle Spectrograph (UVES) at the ESO VLT. Here we present initial results from our ongoing programme to determine temperatures, mass-loss rates and abundances for a large sample of early-type stars in the Clouds.

Optical data from UVES are complemented by ultraviolet spectra from the FUSE hot-star programme and spectra from the HST and IUE archives. We have used the line-blanketed, non-LTE code CMFGEN (Hillier \& Miller 1998) to analyse wind and photospheric features simultaneously to derive intrinsic parameters for nine supergiants in the LMC/SMC (see Crowther et al. 2002, for a full description of our methods).

\section{Results}

For the majority of our targets we find significantly (15-20\%) lower temperatures than those derived from standard plane-parallel, photospheric studies (see Figure 1), highlighting the need for revision of published O-type star temperature 


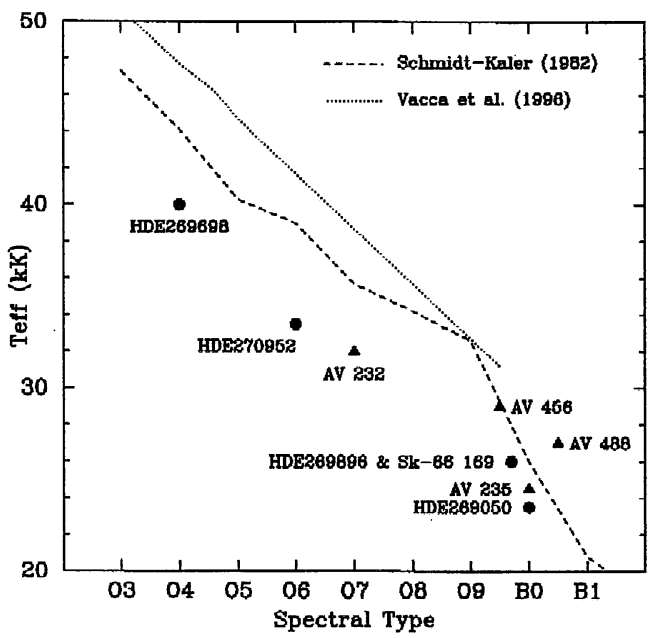

Figure 1. Comparison of derived temperatures with selected calibrations for our current sample of LMC (circles) and SMC (triangles) supergiants.

calibrations (as also demonstrated by, e.g., Bouret et al. and Herrero et al. in these Proceedings). By virtue of the lower temperatures and therefore lower luminosities, these results will clearly have an effect on the wind-momentum luminosity relationship (WLR) for LMC/SMC stars.

The results for AV 456 and AV 488 are of considerable interest as they have much weaker winds than the rest of the sample. Published temperature calibrations are generally monotonic with lower $T_{\text {eff }}$ for later types; however, our result for AV 488 ( $c f$. AV 235 / HDE 269050) illustrates the dependence of stellar temperatures on wind density in addition to spectral type.

Finally, the derived nitrogen mass fractions, $\epsilon(\mathrm{N})$, range from 1.0-9.0 $\epsilon\left(\mathrm{N}_{\odot}\right)$. In the context of the reduced metallicity of the Clouds, these abundances represent significant enhancements. Our results also indicate moderate carbon and oxygen depletions, though not substantial enough to account for the high levels of nitrogen enrichment, if the total CNO abundance is maintained within the CNO-cycle.

\section{References}

Crowther, P.A., Hillier, D.J., Evans, C.J., Fullerton, A.W., De Marco, O., Willis, A.J. 2002b, ApJ 579, 774

Hillier, D.J., Miller, D.L. 1998, ApJ 496, 407

Schmidt-Kaler, T., 1982, in K. Schaifers \& H.H. Voigt (eds.), Landolt-Börnstein, Group VI, Vol 2b (Berlin: Springer), p.1

Vacca, W.D., Garmany, C.D., Shull, J.M. 1996, ApJ 460, 914 